\title{
Comparison of Genetic Algorithm based Watermarking Techniques using Tournament Selection Approach and Roulette Wheel Approach for Fidelity Optimization
}

\author{
Sachin Goyal and Roopam Gupta \\ Department of Information Technology \\ RGPV, Bhopal, India \\ sachingoyal@rgtu.net,ropamgupta@rgtu.net
}

\begin{abstract}
To protect digital content from illegal copy or reproduction, digital watermarking techniques are used which embed watermark into digital content and extract the same from the digital content to help in digital media protection. As techniques based on spatial and frequency domain are reported to have several limitations due to unsatisfactory values of fidelity by various researchers, new techniques based on genetic algorithm have been developed with an objective to optimize the values of fidelity of watermarked image. Genetic algorithms are used to find suitable locations for watermark insertion within a cover image, using either tournament selection approach or roulette wheel selection approach to provide optimization of fidelity. This paper is an attempt to provide a comparative study of the results obtained with genetic based watermarking techniques using roulette wheel selection approach and tournament selection approach. The variation of maximum fitness with respect to changing embedding strength, number of genes, mutation probability. Crossover probability and varying payloads has been compared and discussed for both the selection strategies.
\end{abstract}

Keywords: Digital watermarking, genetic algorithm, fidelity, PSNR, roulette-wheel selection, tournament selection

\section{Introduction}

There are many watermarking methods [1] which have been developed to embed a watermark into the cover image. Some of the important contributions of various proposed methods of digital watermarking during initial research period in digital watermarking are presented here. A watermark was generated using the least significant bit of the original image by Schyndel et al. [2] to produce the watermarked image. The watermark was extracted from a suspected image by taking the least significant bits at the proper locations. Cross-correlation of the original and extracted watermark was made by Schyndel, which showed that the resulting image contained an invisible watermark with simple extraction procedures. Cox et al. [3] pointed that, in order for a watermark to be robust to attack, it must be placed in perceptually significant areas of the image. Yen and Huey et al [4] chose pair of position with same quantization scale in standard JPEG quantization table as cover image and it use the concept of discrete cosine Transformation. Huang et al. [5] paper used a concept of DCT and proposed a Progressive watermarking Techniques with genetic algorithm. M.J Anwar et al. [6] this method is more robust against JPEG compression. In this method genetic algorithm is used to find best position in image block that the positions have a mathematical relationship. Somying promcharoen and yuttapong rangsanseri [7] this approach used a fuzzy C-mean algorithm to classify 8x8 DCT block as texture or non-texture region. In this paper, a digital image watermarking by using genetic algorithm to optimize parameters used in block based DCT watermark embedding. M. Rafig [8] have proposed 
a watermarking algorithm in the DCT domain using an evolutionary algorithm. S. Goyal et al. [9 10] use a roulette-wheel and tournament selection strategy in genetic algorithm and optimize the fidelity in spatial domain. This paper provides comparative study of the results obtained with genetic based watermarking techniques using roulette wheel selection [9] approach and tournament selection approach [10].

Section II provides the methodology adopted with roulette wheel selection and Tournament Selection approach for fidelity optimization. Section III show Experimental Result and Compare the algorithm which was proposed in earlier papers [9 10$]$. Conclusion is given in section IV.

\section{Roulette-Wheel Approach and Tournament Selection Approach for Fidelity optimization}

The purpose of selection is to stress fitter individuals in the population in hope that their off springs have higher fitness. Chromosomes are selected from the initial population to be parents for reproduction. This approach work as follows:

1. Initial population consisting of random location for watermark insertion is chosen.

2. Fitness is designed in such a way that higher fidelity, the higher shall be the value of fitness function.

3. Best individual are select using either Roulette-wheel or tournament selection strategy

4. Now, the crossover and mutation is done to produce new set of locations i.e. new population.

5. Step from 2 to 4 are repeated till termination condition is obtained.

The Principal of Roulette-wheel [11-12] selection is a linear search and it can be visualize similar to roulette-wheel in a casino. Usually a proportion of the wheel is assigned to each of the possible selected based on their fitness of a selection by the total fitness of all the selection.

Tournament Selection [11-12] is a method of selecting an individual from a population. Tournament selection involves running numerous "tournament" among a few individuals chosen at random from population. The victor of each tournament is selected for crossover.

\section{Experimental Result and Comparison}

Roulette-wheel and Tournament selection strategy [9-10] is used to find out the desired locations for watermark insertion for fidelity optimization. In our experiments, the cover images used is Lena size (512 X 512) shown in figure 1 and the binary two dimensional matrix with varying length in different experiments as the watermark. Experimental parameter are given as papers [9-10]

\section{Experiment 1}

Thus it is clear from table 1 and figure 2 to 5 that the highest value of maximum fitness obtained is 78.1907 with the number of watermark bits $=16$ and number of genes $=80$ with embedding strength $=0.1$ in case of tournament selection strategy and the highest value of maximum fitness obtained is 73.4850 with no of watermark bits $=16$ and number of genes $=80$ with embedding strength $=0.1$ in case of roulette wheel selection method. 


\begin{tabular}{|c|c|c|c|c|c|c|c|c|c|c|}
\hline \multicolumn{11}{|c|}{ Table 1. Comparative Table of Variation of Maximum Fitness Vs Embedding Strengths } \\
\hline \multirow{3}{*}{$\begin{array}{c}\text { Waterma } \\
\text { rk Bits/ } \\
\text { No. 0f } \\
\text { Genes } \\
\\
16 / 80\end{array}$} & \multicolumn{2}{|c|}{$\begin{array}{c}\text { Maximum Fitness } \\
\text { when Embedding } \\
\text { Strengths }=0.1\end{array}$} & \multicolumn{2}{|c|}{$\begin{array}{l}\text { Maximum Fitness when } \\
\text { Embedding Strengths }=0.2\end{array}$} & \multicolumn{2}{|c|}{$\begin{array}{c}\text { Maximum Fitness } \\
\text { when Embedding } \\
\text { Strengths }=0.3\end{array}$} & \multicolumn{2}{|c|}{$\begin{array}{c}\text { Maximum Fitness when } \\
\text { Embedding } \\
\text { Strengths }=0.4\end{array}$} & \multicolumn{2}{|c|}{$\begin{array}{c}\text { Maximum Fitness when } \\
\text { Embedding } \\
\text { Strengths }=0.5\end{array}$} \\
\hline & $\begin{array}{l}\text { Tournament } \\
\text { Selection }\end{array}$ & $\begin{array}{l}\text { Roulette } \\
\text { Wheel }\end{array}$ & $\begin{array}{l}\text { Tournament } \\
\text { Selection }\end{array}$ & $\begin{array}{l}\text { Roulette } \\
\text { Wheel }\end{array}$ & $\begin{array}{l}\text { Tournament } \\
\text { Selection }\end{array}$ & $\begin{array}{c}\text { Roulette } \\
\text { Wheel }\end{array}$ & $\begin{array}{l}\text { Tournament } \\
\text { Selection }\end{array}$ & $\begin{array}{c}\text { Roulette } \\
\text { Wheel }\end{array}$ & $\begin{array}{l}\text { Tournament } \\
\text { Selection }\end{array}$ & $\begin{array}{c}\text { Roulette } \\
\text { Wheel }\end{array}$ \\
\hline & 78.1907 & 73.4850 & 74.2013 & 68.2695 & 68.1523 & 63.5633 & 64.4774 & 61.2810 & 64.3328 & 58.6833 \\
\hline $20 / 100$ & 75.9836 & 72.3213 & 72.3153 & 66.2614 & 66.3448 & 62.0214 & 65.7418 & 59.8865 & 60.7591 & 58.2793 \\
\hline $24 / 120$ & 74.4120 & 71.5851 & 68.6929 & 65.1345 & 65.7568 & 61.1399 & 64.3524 & 59.0840 & 61.3901 & 57.0121 \\
\hline $28 / 140$ & 73.6137 & 69.6006 & 68.8274 & 64.3120 & 63.2830 & 60.2322 & 61.7785 & 57.6576 & 60.3328 & 56.8408 \\
\hline $32 / 160$ & 71.9436 & 68.9753 & 65.4888 & 63.9612 & 62.6579 & 60.5623 & 60.2618 & 56.4570 & 57.4112 & 55.9521 \\
\hline $40 / 200$ & 71.7780 & 68.3329 & 64.9562 & 62.1386 & 60.4088 & 59.1342 & 59.5264 & 55.8521 & 56.4498 & 55.0198 \\
\hline $56 / 280$ & 69.027 & 66.7266 & 62.7078 & 62.2650 & 60.2377 & 57.3567 & 57.8788 & 54.2031 & 55.6938 & 53.9854 \\
\hline $112 / 560$ & 66.2833 & 65.6401 & 61.2312 & 59.6134 & 56.3472 & 56.2511 & 54.2084 & 53.8268 & 52.5103 & 51.8345 \\
\hline $128 / 640$ & 65.1021 & 65.3751 & 60.9102 & 59.1463 & 56.1604 & 55.4706 & 53.6875 & 53.1240 & 51.6456 & 51.2231 \\
\hline $\begin{array}{c}256 / 128 \\
0\end{array}$ & 64.7346 & 64.7660 & 58.7120 & 58.2441 & 55.1922 & 55.1922 & 52.9507 & 52.6525 & 50.7522 & 50.4143 \\
\hline
\end{tabular}

The maximum value of fidelity varies between 78.1907 to 64.3328 for tournament selection method when embedding strength varies form 0.1 to 0.5 and varies from 73.4850 to 58.6833 in case of roulette wheel selection method when embedding strength varies from 0.1 to 0.5 .

Thus it can be inferred from this experiment that the fidelity obtained with tournament selection strategy has an edge over the fidelity obtained with roulette wheel strategy with changing values of embedding strength for a specific value of no of genes and number of watermark bits.

It is also observed from table 1 that with less no of watermarking bits / no of genes, the value of maximum fidelity obtained with tournament selection strategy is much better as compared to roulette wheel selection strategy. However, with increasing number of watermark bits and number of genes, this difference goes on diluting. Figure 2 (a) to (d) shows variation of maximum fitness with respect to changing Embedding Strength.

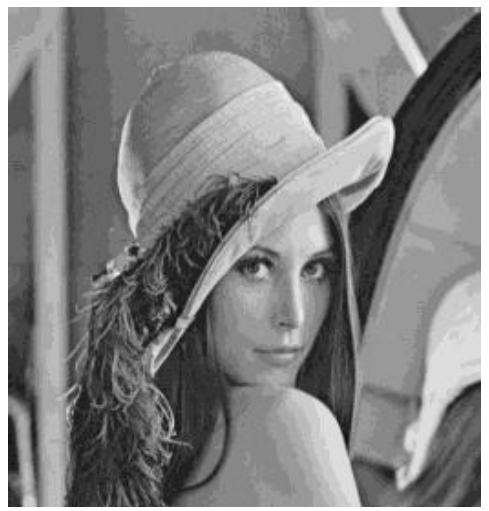

Figure 1. Lena Image $(512 \times 512)$ 


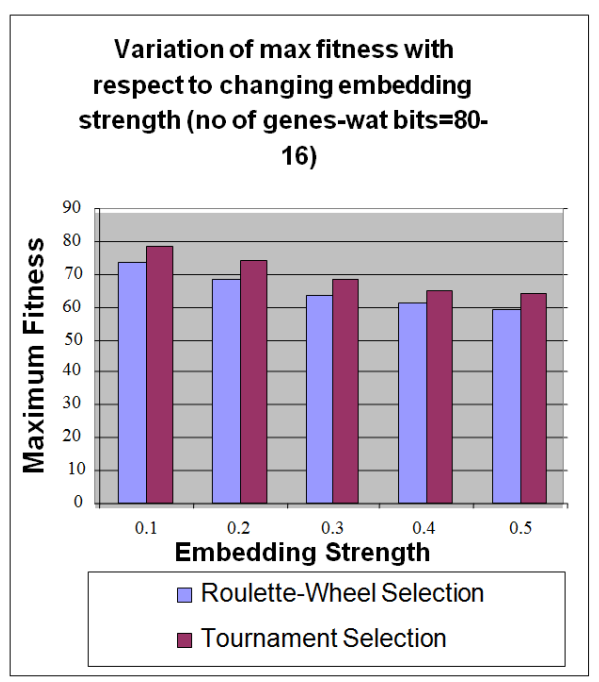

(a)

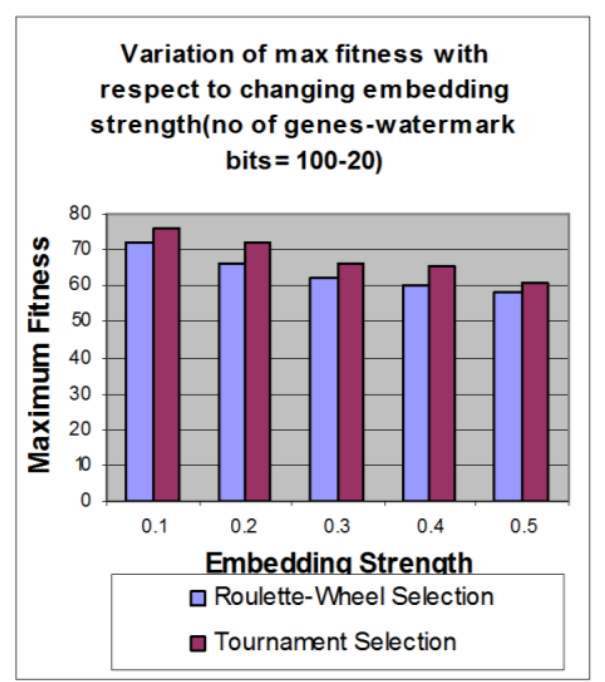

(c)

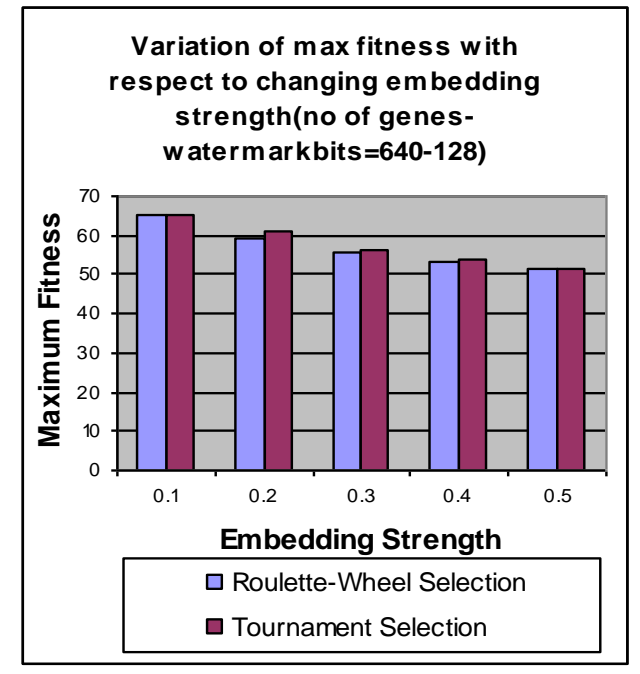

(b)

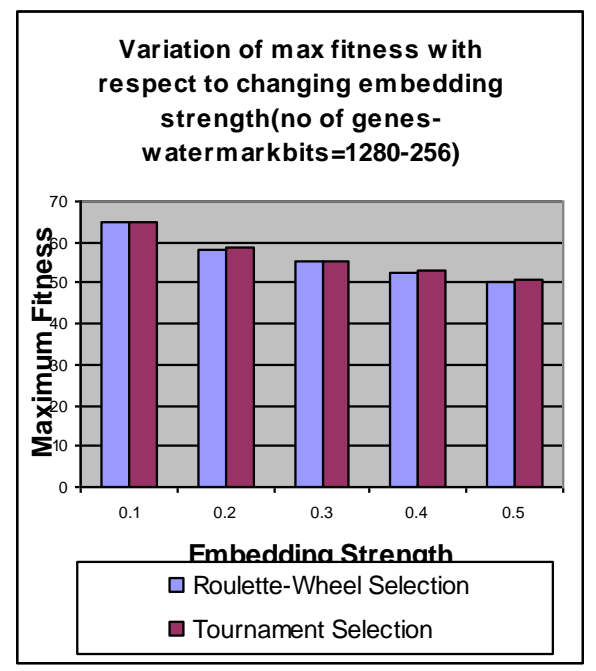

(d)

Figure 2. (a) - (d) Variation of Maximum Fitness with Embedding Strength

\section{Experiment 2}

Thus it is clear from table 2 that the highest value of maximum fitness obtained is 64.6275 with the number of watermark bits $=16$ and number of genes $=80$ with crossover probability $=0.85$ in case of tournament selection strategy and the highest value of maximum fitness obtained is 59.0554 with no of watermark bits $=16$ and number of genes $=80$ with crossover probability $=0.85$ in case of roulette wheel selection method.

The maximum value of fidelity varies between 64.6275 to 62.2245 for tournament selection method when crossover probability varies form 0.85 to 0.35 and varies from 59.0554 to 58.4439 of roulette wheel selection method when crossover probability varies form 0.85 to 0.35 .

Thus it can be inferred from this experiment that the fidelity obtained with tournament selection strategy has an edge over the fidelity obtained with roulette wheel strategy with changing values of crossover probability for a specific value of no of genes and number of watermark bits. Figure 3(a) and (b) shows Variation of maximum fitness with respect to changing crossover probability. 


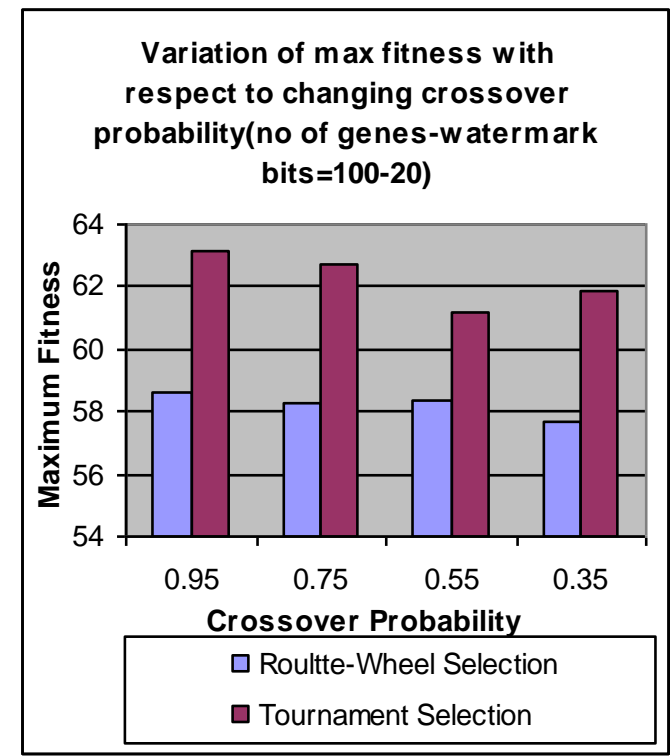

(a) (b)

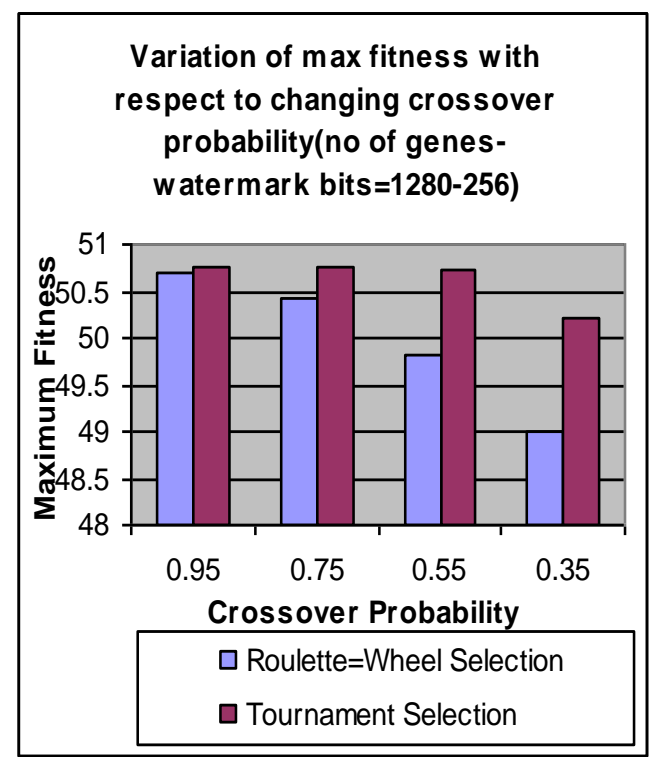

(b)

Figure 3. (a) and (b) Variation of Maximum Fitness with Crossover Probability

\section{Experiment 3}

Thus it is clear from table 3 that the highest value of maximum fitness obtained is 65.4005 with the no of watermark bits $=16$ and number of genes $=80$ with mutation probability $=0.001$ in case of tournament selection strategy and the highest value of maximum fitness obtained is 59.1461 with no of watermark bits $=16$ and number of genes $=80$ with mutation probability $=0.001$ in case of roulette wheel selection method.

The maximum value of fidelity varies between 65.4005 to 60.4405 for tournament selection method when mutation probability varies form 0.001 to 0.1 and varies from 59.1462 to 58.0720 of roulette wheel selection method when mutation probability varies form 0.001 to 0.1 .

Thus it can be inferred from this experiment that the fidelity obtained with tournament selection strategy has an edge over the fidelity obtained with roulette wheel strategy with changing values of mutation probability for a specific value of no of genes and number of watermark bits.

It is also observed from table 3 that with less no of watermarking bits / no of genes, the value of maximum fidelity obtained with tournament selection strategy is much better as compared to roulette wheel selection strategy.

Thus, with higher no of watermark bits, the results produced by both the selection strategies are quite similar. Figure 4(a) and (b) shows variation of maximum fitness 


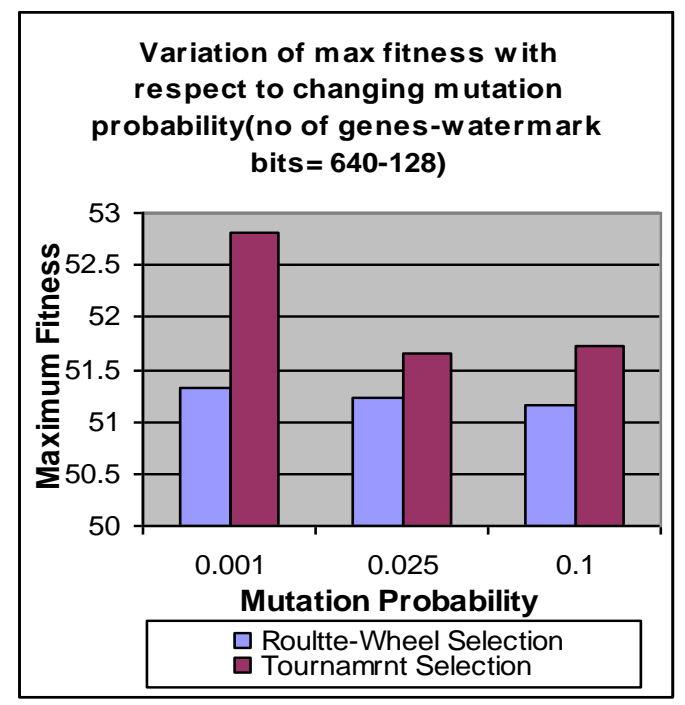

(a)

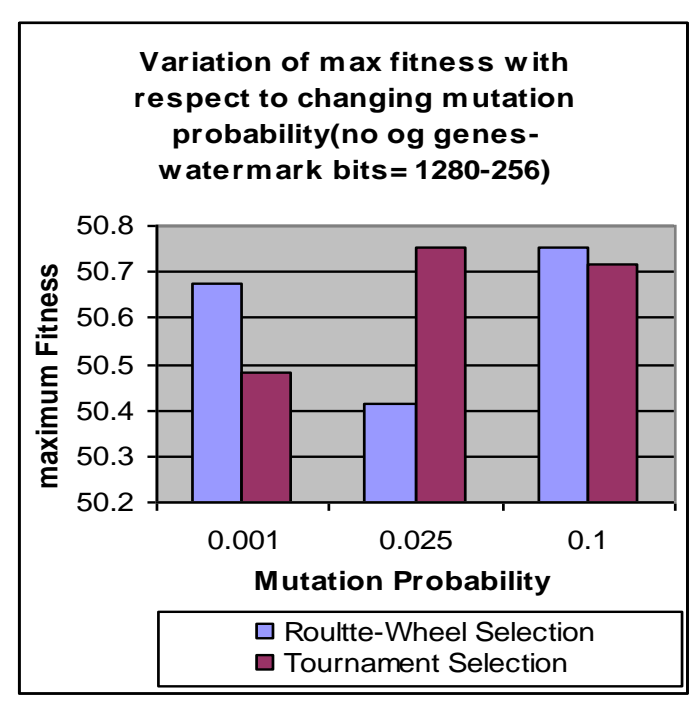

(b)

Figure 4. (a) and (b) Variation of Maximum Fitness with Mutation Probability

Table 3. Comparative Table of Maximum Fitness Vs Mutation Probability

\begin{tabular}{|c|c|c|c|c|c|c|}
\hline $\begin{array}{c}\text { Watermark } \\
\text { Bits/ } \\
\begin{array}{c}\text { No. 0f } \\
\text { Genes }\end{array}\end{array}$ & \multicolumn{2}{|c|}{$\begin{array}{c}\text { Maximum Fitness when } \\
\text { Mutation probability=0.001 }\end{array}$} & \multicolumn{2}{c|}{$\begin{array}{c}\text { Maximum Fitness when } \\
\text { Mutation Probability =0.025 }\end{array}$} & \multicolumn{2}{c|}{$\begin{array}{c}\text { Maximum Fitness when } \\
\text { Mutation Probability }=0.1\end{array}$} \\
\cline { 2 - 7 } & $\begin{array}{c}\text { Tournament } \\
\text { Selection }\end{array}$ & $\begin{array}{c}\text { Roulette } \\
\text { Wheel }\end{array}$ & $\begin{array}{c}\text { Tournament } \\
\text { Selection }\end{array}$ & $\begin{array}{c}\text { Roulette } \\
\text { Wheel }\end{array}$ & $\begin{array}{c}\text { Tournament } \\
\text { Selection }\end{array}$ & $\begin{array}{c}\text { Roulette } \\
\text { Wheel }\end{array}$ \\
\hline $16 / 80$ & 65.4005 & 59.1461 & 64.3328 & 58.6833 & 60.4405 & 58.0720 \\
\hline $20 / 100$ & 61.2048 & 58.4750 & 60.7591 & 58.2793 & 59.4490 & 58.1832 \\
\hline $24 / 120$ & 62.1927 & 57.9520 & 61.3901 & 57.0121 & 59.1827 & 56.6025 \\
\hline $28 / 140$ & 61.3076 & 56.5310 & 60.3328 & 56.8408 & 56.6026 & 55.9825 \\
\hline $32 / 160$ & 58.6093 & 56.5289 & 57.4112 & 55.8603 & 55.9508 & 55.2598 \\
\hline $40 / 200$ & 58.5565 & 56.2124 & 56.4498 & 55.0198 & 55.0841 & 54.3355 \\
\hline $56 / 280$ & 56.4077 & 54.1998 & 55.6938 & 53.9854 & 53.3143 & 53.1354 \\
\hline $112 / 560$ & 55.0254 & 52.2860 & 52.5103 & 51.8345 & 52.1111 & 51.4612 \\
\hline $128 / 640$ & 52.8031 & 51.3112 & 51.6456 & 51.2231 & 51.7361 & 51.1474 \\
\hline $256 / 1280$ & 50.4836 & 50.6723 & 50.7522 & 50.4143 & 50.7143 & 50.2522 \\
\hline
\end{tabular}

\section{Conclusions}

This paper has presented a comparative study of the variations in maximum fidelity obtained using watermarking techniques with genetic algorithms using roulette wheel selection method and tournament selection method. The results obtained from both these selection with respect to changing crossover probability. Obtained between the two selection strategies with different settings of the number of genes, crossover probability, Mutation probability, embedding strengths and payloads etc. This work can serve as the basis of using a specific selection strategy for the fidelity optimization with a given set of parameters.

\section{References}

[1] SachinGoyal, R.Gupta, A.Bansal "Survey of Digital Watermarking with Genetic Algorithm" in Computer Society of India Communication Jan-2010.pp22-25.

[2] R.G.Van Schyndel,A.Z.Tirkel and CF.Osborene, "A Digital Watermark" in Proc. IEEE International Conf. Image processing,1994, vol.2 pp 86-92. 
[3] J.Cox,J.Kilian, "A Secure Robust Watermark for Multimedia” in Proc. First International Workshop, vol 1174 of Lecture notes in computer science, pp. 185-206.

[4] Yen, Shwu Huey, et al. “ A Watermarking Technique based on JPEG quantization table.” Taipei, Taiwan: Proceeding of the 2006 International Computer Symposium (ICS2006).

[5] Huang ,Pan, Shieh and Wang "Progressive watermarking techniques with genetic Algorithms" IEEE, 2003.

[6] M.J. Anwar, M.Ishtiaq, M.Iqqbal, M,Jaffar “ Blocked-based digital image watermarking using Genetic Algorithm" in Proc IEEE international conference on Emerging Technologies(ICET) 2010. pp 204-208.

[7] Somying Promchareon and Yuttapong Rangsanseri. "Genetic watermarking with Block-Based DCT Clustering in IEEE International Symposium on Communication and Information Technologies(ISCIT2008). Pp 346-351

[8] M.rafigh and M.Ebrahimi “ A Robust Evolutionary Based Digital Image Watermarking Technique in DCT Domain" in Proc IEEE Seventh International Conference on Computer Graphics, Imaging and Visualization 2010.pp 105-109.

[9] Sachin Goyal and Roopam Gupta "Optimization of fidelity with Adaptive genetic watermarking algorithm using roulette-wheel" in proc IEEE International conf. On Computational Intelligence Communication Networls", 2010. pp 591-596.

[10] Sachin Goyal and Roopam Gupta "Optimization of Fidelity with Adaptive Genetic Watermarking Algorithm using Tournament Selection” International Journal of Advanced Science and Technology Vol. 30, May, 2011 pp 55-65.

[11] Melanie Mitchell “ An Introduction to Genetic Algorithm” MIT Press Edition 1998.

[12] M.Srinivas and Lalit M.Patnaik, "Genetic Algorithm: A Survey” IEEE,1994. 
International Journal of Security and Its Applications

Vol.9, No.10 (2015) 